\title{
Large-time Behavior of Magnetohydrodynamics with Temperature-Dependent Heat-Conductivity *
}

\author{
Bin Huang ${ }^{1}$, Xiaoding Shi ${ }^{1}$, Ying Sun ${ }^{\dagger} \dagger$ \\ 1. Department of Mathematics, College of Mathematics and Physics, \\ Beijing University of Chemical Technology, \\ Beijing 100029, P. R. China \\ 2. School of Mathematical Sciences, Xiamen University, \\ Xiamen 361005, P. R. China
}

\begin{abstract}
We study the large-time behavior of strong solutions to the equations of a planar magnetohydrodynamic compressible flow with the heat conductivity proportional to a nonnegative power of the temperature. Both the specific volume and the temperature are proved to be bounded from below and above independently of time. Moreover, it is shown that the global strong solution is nonlinearly exponentially stable as time tends to infinity. Our result can be regarded as a natural generalization of the previous ones for the compressible Navier-Stokes system to MHD system with either constant heat-conductivity or nonlinear and temperaturedependent heat-conductivity.
\end{abstract}

Keywords. Magnetohydrodynamics, Temperature-dependent heat-conductivity, Strong solutions, Large-time behavior

Math Subject Classification: 35Q35; 76N10.

\section{Introduction}

The governing equations of a planar magnetohydrodynamic compressible flow written in the Lagrange variables read as follows:

$$
\begin{gathered}
v_{t}=u_{x}, \\
u_{t}+\left(P+\frac{1}{2}|\mathbf{b}|^{2}\right)_{x}=\left(\mu \frac{u_{x}}{v}\right)_{x}, \\
\mathbf{w}_{t}-\mathbf{b}_{x}=\left(\lambda \frac{\mathbf{w}_{x}}{v}\right)_{x}, \\
(v \mathbf{b})_{t}-\mathbf{w}_{x}=\left(\nu \frac{\mathbf{b}_{x}}{v}\right)_{x},
\end{gathered}
$$

${ }^{*}$ Partially supported by NNSFC 11671027 and 11471321.

${ }^{\dagger}$ Email addresses: abinhuang@gmail.com, abinhuang36@163.com (B. Huang), shixd@mail.buct.edu.cn (X. Shi) 1913349041@qq.com (Y. Sun) 


$$
\begin{aligned}
& \left(e+\frac{u^{2}+|\mathbf{w}|^{2}+v|\mathbf{b}|^{2}}{2}\right)_{t}+\left(u\left(P+\frac{1}{2}|\mathbf{b}|^{2}\right)-\mathbf{w} \cdot \mathbf{b}\right)_{x} \\
& =\left(\kappa \frac{\theta_{x}}{v}+\mu \frac{u u_{x}}{v}+\lambda \frac{\mathbf{w} \cdot \mathbf{w}_{x}}{v}+\nu \frac{\mathbf{b} \cdot \mathbf{b}_{x}}{v}\right)_{x},
\end{aligned}
$$

where $t>0$ is time, $x \in \Omega=(0,1)$ denotes the Lagrange mass coordinate, and the unknown functions $v>0, u, \mathbf{w} \in \mathbb{R}^{2}, \mathbf{b} \in \mathbb{R}^{2}, e>0, \theta>0$, and $P$ are, respectively, the specific volume of the gas, longitudinal velocity, transverse velocity, transverse magnetic field, internal energy, absolute temperature and pressure. $\mu$ and $\lambda$ are the viscosity of the flow, $\nu$ is the magnetic diffusivity of the magnetic field, and $\kappa$ is the heat conductivity.

In this paper, we consider a perfect gas for magnetohydrodynamic flow, that is, $P$ and $e$ satisfy

$$
P=R \theta / v, \quad e=c_{v} \theta+\text { const, }
$$

where both specific gas constant $R$ and heat capacity at constant volume $c_{v}$ are positive constants. We also assume that $\mu, \lambda$, and $\nu$ are positive constants, and $\kappa$ satisfies

$$
\kappa=\tilde{\kappa} \theta^{\beta},
$$

with constants $\tilde{\kappa}>0$ and $\beta \geq 0$.

The system (1.1)-(1.7) is supplemented with initial conditions

$$
(v, u, \theta, \mathbf{b}, \mathbf{w})(x, 0)=\left(v_{0}, u_{0}, \theta_{0}, \mathbf{b}_{0}, \mathbf{w}_{0}\right)(x), \quad x \in \Omega,
$$

and boundary ones

$$
\left.\left(u, \mathbf{b}, \mathbf{w}, \theta_{x}\right)\right|_{\partial \Omega}=0,
$$

where the initial data (1.8) should be compatible with the boundary conditions (1.9).

Magnetohydrodynamics (MHD), concerning the flow of electrically conducting fluids in the presence of magnetic fields, covers a wide range of physical objects from liquid metals to cosmic plasmas ( 6 , 10, 17, 19, 23, 24, 31]). The central point of MHD theory is that conductive fluids can support magnetic fields. The partial differential equations of MHD can in principle be derived from Boltzmann's equation assuming space and time scales to be larger than all inherent scale-lengths such as the Debye length or the gyro-radii of the charged particles ( $66,17,23,24,31)$. In fact, one can deduce from the Chapman-Enskog expansion for the first level of approximation in kinetic theory that the viscosity $\mu$ and heat conductivity $\kappa$ are functions of temperature alone (see Chapman-Colwing [8]). These dependencies, especially the dependence of viscosity on temperature, brings great difficulties and challenges to mathematical analysis and numerical calculation. Thus, to study this problem, we first consider the case that the viscosity is a positive constant and the heat conductivity proportional to a nonnegative power of the temperature, as shown as in the equation (1.7).

There is huge literature on the studies of the global existence and large time behavior of solutions to the compressible Navier-Stokes system and MHD system. Indeed, for compressible Navier-Stokes system (1.1) (1.2) (1.5) with $\mathbf{b} \equiv \mathbf{w} \equiv 0$, Kazhikhov and Shelukhin [22] first obtained the global existence of solutions for constant coefficients $(\beta=0)$ with large initial data. From then on, much effort has been made to generalize this approach to other cases (for $\beta>0$, see [15, 18, 30] and the reference therein). As for MHD system, the are many results concerning the global existence of solutions with large initial data (see [2,7,9,11, 14, 16, 21,33] and the references therein). In particular, Kazhikhov 21] (see also 2]) first for $\beta=0$ and very recently Huang-Shi-Sun [16] for $\beta>0$ proved that 
Lemma 1.1 ( [16, 21] $)$. Let $\beta \geq 0$. Suppose that the initial data $\left(v_{0}, u_{0}, \theta_{0}, \mathbf{b}_{0}, \mathbf{w}_{0}\right)$ satisfies

$$
\left(v_{0}, \theta_{0}\right) \in H^{1}(0,1), \quad\left(u_{0}, \mathbf{b}_{0}, \mathbf{w}_{0}\right) \in H_{0}^{1}(0,1),
$$

and

$$
\inf _{x \in(0,1)} v_{0}(x)>0, \quad \inf _{x \in(0,1)} \theta_{0}(x)>0 .
$$

Then, the initial-boundary-value problem (1.1)-(1.9) has a unique strong solution $(v, u, \theta$, $\mathbf{b}, \mathbf{w})$ such that for each fixed $T>0$,

$$
\left\{\begin{array}{l}
v, \theta \in L^{\infty}\left(0, T ; H^{1}(0,1)\right), \quad u, \mathbf{b}, \mathbf{w} \in L^{\infty}\left(0, T ; H_{0}^{1}(0,1)\right), \\
v_{t} \in L^{\infty}\left(0, T ; L^{2}(0,1)\right) \cap L^{2}\left(0, T ; H^{1}(0,1)\right), \\
u_{t}, \theta_{t}, \mathbf{b}_{t}, \mathbf{w}_{t}, u_{x x}, \theta_{x x}, \mathbf{b}_{x x}, \mathbf{w}_{x x} \in L^{2}((0,1) \times(0, T)),
\end{array}\right.
$$

and for each $(x, t) \in[0,1] \times[0, T]$

$$
C^{-1} \leq v(x, t) \leq C, \quad C^{-1} \leq \theta(x, t) \leq C,
$$

where $C>0$ is a constant depending on the data and $T$.

Concerning the large-time behavior of solutions to the compressible Navier-Stokes system (1.1) (1.2) (1.5) with $\mathbf{b} \equiv \mathbf{w} \equiv 0$, Kazhikhov [20] (see also [1, 3- 5, 25, 28, 32 among others) first obtained that for the case that $\beta=0$, the strong solution is nonlinearly exponentially stable as time tends to infinity. Very recently, Huang-Shi [15] prove that the same result still holds for the compressible Navier-Stokes system (1.1) (1.2) (1.5) with $\mathbf{b} \equiv \mathbf{w} \equiv 0$ for $\beta>0$. However, it seems to us that the known lower and upper bounds of the the specific volume $v$ and the temperature $\theta$ depend on the time $T$, see [16, 21], so it is impossible to study the large time asymptotic behavior of solutions in the setting in [16, 21. In fact, the main aim of this paper is to prove that the global strong solutions whose existence is guaranteed by Lemma 1.1 are nonlinearly exponentially stable as time tends to infinity for $\beta \geq 0$.

We now state our main result as follows.

Theorem 1.2. Under the conditions of Lemma 1.1, there exist positive constants $C$ and $\eta_{0}$ both depending only on the data such that the unique strong solution $(v, u, \theta, \mathbf{b}, \mathbf{w})$ of the initial-boundary-value problem (1.1)-(1.9) obtained by Lemma 1.1 satisfies for any $(x, t) \in(0,1) \times(0, \infty)$,

$$
C^{-1} \leq v(x, t) \leq C, \quad C^{-1} \leq \theta(x, t) \leq C,
$$

and for any $t>0$,

$$
\left\|\left(v-v_{s}, u, \theta-\theta_{s}, \mathbf{b}, \mathbf{w}\right)\right\|_{H^{1}(0,1)} \leq C e^{-\eta_{0} t},
$$

with

$$
v_{s}=\int_{0}^{1} v_{0} d x, \quad \theta_{s}=\int_{0}^{1}\left(\theta_{0}+\frac{u_{0}^{2}+\left|\mathbf{w}_{0}\right|^{2}+v_{0}\left|\mathbf{b}_{0}\right|^{2}}{2 c_{v}}\right) d x
$$

Remark 1.1. Our result can be regarded as a natural generalization of previous ones for compressible Navier-Stokes system with either constant heat conductivity ([20]) or temperature-dependent one ([15]) to the temperature-dependent heat conductivity MHD system with the constant heat conductivity as a special case. 
We now make some comments on the analysis of this paper. The key step to study the large-time behavior of the global strong solutions is to get the time-independent lower and upper bounds of both $v$ and $\theta$ (see (2.1), (2.18), (2.50), and (2.95)). Compared with [15, 20,28], the main difficulties come from the interaction of the hydrodynamic and electrodynamic effects and the degeneracy and nonlinearity of the heat conductivity. Hence, to overcome these difficulties, some new ideas are needed. The key observations are as follows: First, after modifying the ideas due to [15, 20], we obtain an explicit expression of the specific volume $v$ (see (2.9) $)$ which together with a lower bound of the temperature $\theta$ (see (2.15) ) shows that $v$ is bounded from below time-independently (see (2.1) ). Then, for $\beta>0$, we find that (see (2.19) )

$$
\int_{0}^{T} \max _{x \in[0,1]}\left(\theta^{1 / 2}(x, t)-2\right)_{+}^{2} d t \leq C
$$

which gives the uniform upper bound of $v$. For $\beta=0$, it seems much more difficult to bound $v$ from above time-independently. We first prove a new estimate that (see (2.34) )

$$
\int_{0}^{T} \int_{0}^{1} \frac{\left|\mathbf{w}_{x}\right|^{2}}{v} d x d t \leq C
$$

which play an important role in the analysis. Then we refine the strategy of Kazhikhov ( [21]), that is, we prove that the $L^{\infty}\left(0, T ; L^{2}(0,1)\right)$-norm of $(\ln v)_{x}$ can be bounded timeindependently by a log-type inequality (see (2.49)), which together with the Gronwall inequality in turn gives the uniform upper bound of $v$ (see (2.50) ). Next, for $\beta>0$ and for the upper and lower bounds of $\theta$, we modify slightly the ideas due to [15], that is, we prove that the $L^{\infty}\left(0, \infty ; L^{p}\right)$-norm of $\theta^{-1}$ is bounded (see (2.25)), which yields that the $L^{2}((0,1) \times(0, T))$-norm of $\theta_{x}$ is bounded provided $\beta>1$ (see (2.79)). Finally, for $\beta \in[0,1]$, we find that the $L^{2}((0,1) \times(0, T))$-norm of $\theta_{x}$ can be bounded by the $L^{4}\left(0, T ; L^{2}(0,1)\right)$-norm of $u_{x}$ which plays an important role in obtaining the uniform bound on $L^{2}((0,1) \times(0, T))$-norm of both $\theta_{x}$ and $u_{x x}$ (see Lemma 2.7) for $\beta \in[0,1]$. The whole procedure will be carried out in the next section.

\section{$2 \quad$ Proof of Theorem 1.2}

Without loss of generality, we assume that $\lambda=\nu=\mu=\tilde{\kappa}=R=c_{v}=1$, and that

$$
\int_{0}^{1} v_{0} d x=1, \quad \int_{0}^{1}\left(\theta_{0}+\frac{u_{0}^{2}+\left|\mathbf{w}_{0}\right|^{2}+v_{0}\left|\mathbf{b}_{0}\right|^{2}}{2}\right) d x=1 .
$$

We first state the time-independent lower bound of $v$.

Lemma 2.1. For $\beta \geq 0$, it holds that for any $(x, t) \in[0,1] \times[0,+\infty)$,

$$
v(x, t) \geq C_{0},
$$

where (and in what follows) $C_{0}$ and $C$ denote some generic positive constants depending only on $\beta,\left\|\left(v_{0}, u_{0}, \theta_{0}, \mathbf{b}_{0}, \mathbf{w}_{0}\right)\right\|_{H^{1}(0,1)}, \inf _{x \in[0,1]} v_{0}(x)$, and $\inf _{x \in[0,1]} \theta_{0}(x)$.

Proof. First, it follows from (1.1), (1.5), and (1.9) that for $t>0$

$$
\int_{0}^{1} v(x, t) d x \equiv 1, \quad \int_{0}^{1}\left(\theta+\frac{u^{2}+|\mathbf{w}|^{2}+v|\mathbf{b}|^{2}}{2}\right)(x, t) d x \equiv 1 .
$$


Then, denoting

$$
\sigma \triangleq \frac{u_{x}}{v}-\frac{\theta}{v}-\frac{1}{2}|\mathbf{b}|^{2}
$$

we rewrite (1.2) as

$$
u_{t}=\sigma_{x} .
$$

Integrating this with respect to $x$ over $(0, x)$ gives

$$
\left(\int_{0}^{x} u d y\right)_{t}=\sigma-\sigma(0, t)
$$

which implies

$$
v \sigma(0, t)=v \sigma-v\left(\int_{0}^{x} u d y\right)_{t}
$$

Integrating this with respect to $x$ over $(0,1)$, we obtain after using (1.9), (2.2), and (2.3) that

$$
\begin{aligned}
\sigma(0, t)= & \int_{0}^{1}\left(u_{x}-\theta-\frac{v}{2}|\mathbf{b}|^{2}\right) d x-\left(\int_{0}^{1} v \int_{0}^{x} u d y d x\right)_{t} \\
& +\int_{0}^{1} u_{x} \int_{0}^{x} u d y d x \\
= & -\left(\int_{0}^{1} v \int_{0}^{x} u d y d x\right)_{t}-\int_{0}^{1}\left(\theta+\frac{v}{2}|\mathbf{b}|^{2}+u^{2}\right) d x
\end{aligned}
$$

Finally, combining (2.4), (1.1), and (2.5) yields

$$
v(x, t)=D(x, t) Y(t) \exp \left\{\int_{0}^{t}\left(\theta+\frac{v}{2}|\mathbf{b}|^{2}\right) v^{-1} d \tau\right\}
$$

with

$$
\begin{aligned}
D(x, t)= & v_{0} \exp \left\{\int_{0}^{x}\left(u(y, t)-u_{0}(y)\right) d y\right\} \\
& \times \exp \left\{-\int_{0}^{1} v \int_{0}^{x} u d y d x+\int_{0}^{1} v_{0} \int_{0}^{x} u_{0} d y d x\right\},
\end{aligned}
$$

and

$$
Y(t)=\exp \left\{-\int_{0}^{t} \int_{0}^{1}\left(u^{2}+\frac{v}{2}|\mathbf{b}|^{2}+\theta\right) d x d \tau\right\} .
$$

Using (2.6), direct computation gives

$$
v(x, t)=D(x, t) Y(t)\left\{1+\int_{0}^{t} \frac{\left(\theta+\frac{v}{2}|\mathbf{b}|^{2}\right)(x, \tau)}{D(x, \tau) Y(\tau)} d \tau\right\} .
$$

Next, using (1.1)-(1.4), we rewrite the energy equation (1.5) as

$$
\theta_{t}+\frac{\theta}{v} u_{x}=\left(\frac{\theta^{\beta} \theta_{x}}{v}\right)_{x}+\frac{u_{x}^{2}+\left|\mathbf{w}_{x}\right|^{2}+\left|\mathbf{b}_{x}\right|^{2}}{v} .
$$


Multiplying (1.1), (1.2), (1.3), (1.4), and (2.10) by $1-v^{-1}, u, \mathbf{w}, \mathbf{b}$, and $1-\theta^{-1}$ respectively, adding them altogether and integrating the result over $(0,1) \times(0, T)$, we obtain the following energy-type inequality

$$
\begin{aligned}
& \sup _{0 \leq t \leq T} \int_{0}^{1}\left(\frac{u^{2}+|\mathbf{w}|^{2}+v|\mathbf{b}|^{2}}{2}+(v-\ln v)+(\theta-\ln \theta)\right) d x \\
& \quad+\int_{0}^{T} V(s) d s \leq e_{0},
\end{aligned}
$$

where

$$
V(t) \triangleq \int_{0}^{1}\left(\frac{\theta^{\beta} \theta_{x}^{2}}{v \theta^{2}}+\frac{u_{x}^{2}+\left|\mathbf{w}_{x}\right|^{2}+\left|\mathbf{b}_{x}\right|^{2}}{v \theta}\right)(x, t) d x
$$

and

$$
e_{0} \triangleq 2 \int_{0}^{1}\left(\frac{u_{0}^{2}+\left|\mathbf{w}_{0}\right|^{2}+v_{0}\left|\mathbf{b}_{0}\right|^{2}}{2}+\left(v_{0}-\ln v_{0}\right)+\left(\theta_{0}-\ln \theta_{0}\right)\right) d x \text {. }
$$

Next, applying Jensen's inequality to the convex function $\theta-\ln \theta$ leads to

$$
\int_{0}^{1} \theta d x-\ln \int_{0}^{1} \theta d x \leq \int_{0}^{1}(\theta-\ln \theta) d x
$$

which together with (2.11) and (2.2) leads to

$$
\bar{\theta}(t) \triangleq \int_{0}^{1} \theta(x, t) d x \in\left[\alpha_{1}, 1\right]
$$

where $0<\alpha_{1}<\alpha_{2}$ are two roots of

$$
x-\ln x=e_{0} .
$$

Next, both (2.2) and Cauchy's inequality give

$$
\begin{aligned}
\left|\int_{0}^{1} v \int_{0}^{x} u d y d x\right| & \leq \int_{0}^{1} v\left|\int_{0}^{x} u d y\right| d x \\
& \leq \int_{0}^{1} v\left(\int_{0}^{1} u^{2} d y\right)^{1 / 2} d x \\
& \leq C
\end{aligned}
$$

which combined with (2.7) shows

$$
C^{-1} \leq D(x, t) \leq C .
$$

Moreover, one deduces from (2.2) that

$$
\alpha_{1} \leq \int_{0}^{1}\left(u^{2}+\frac{v|\mathbf{b}|^{2}}{2}+\theta\right) d x \leq 2
$$

which yields that for any $0 \leq \tau<t<\infty$,

$$
e^{-2 t} \leq Y(t) \leq 1, \quad e^{-2(t-\tau)} \leq \frac{Y(t)}{Y(\tau)} \leq e^{-\alpha_{1}(t-\tau)} .
$$


Next, denoting $f_{+} \triangleq \max \{f, 0\}$, we have

$$
\begin{aligned}
\left(\bar{\theta}^{\frac{\beta+1}{2}}(t)-\theta^{\frac{\beta+1}{2}}(x, t)\right)_{+} & \leq \int_{0}^{1}\left|\partial_{x}\left(\bar{\theta}^{\frac{\beta+1}{2}}(t)-\theta^{\frac{\beta+1}{2}}(x, t)\right)_{+}\right| d x \\
& \leq C\left(\int_{0}^{1} \frac{\theta^{\beta} \theta_{x}^{2}}{\theta^{2} v} d x\right)^{1 / 2}\left(\int_{0}^{1} 1_{(\theta \leq \bar{\theta})} \theta v d x\right)^{1 / 2} \\
& \leq C V^{1 / 2}(t),
\end{aligned}
$$

which implies that for $t>0$,

$$
\min _{x \in[0,1]} \theta(x, t) \geq \frac{\alpha_{1}}{4}-C V(t) .
$$

Combining (2.13)-(2.15) yields that

$$
\begin{aligned}
v(x, t) & \geq C^{-1} \int_{0}^{t} e^{-2(t-\tau)} \min _{x \in[0,1]} \theta(x, \tau) d \tau \\
& \geq C^{-1} \int_{0}^{t} e^{-2(t-\tau)}\left(\frac{\alpha_{1}}{4}-C V(\tau)\right) d \tau \\
& \geq \frac{C^{-1} \alpha_{1}}{8}-\frac{C^{-1} \alpha_{1}}{8} e^{-2 t}-C \int_{0}^{t} e^{-2(t-\tau)} V(\tau) d \tau .
\end{aligned}
$$

Since

$$
\begin{aligned}
\int_{0}^{t} e^{-2(t-\tau)} V(\tau) d \tau & =\int_{0}^{t / 2} e^{-2(t-\tau)} V(\tau) d \tau+\int_{t / 2}^{t} e^{-2(t-\tau)} V(\tau) d \tau \\
& \leq e^{-t} \int_{0}^{\infty} V(\tau) d \tau+\int_{t / 2}^{t} V(\tau) d \tau \rightarrow 0, \text { as } t \rightarrow \infty
\end{aligned}
$$

it follows from (2.16) that there exists some $\tilde{T}>0$ such that

$$
v(x, t) \geq \frac{C^{-1} \alpha_{1}}{16}
$$

for all $(x, t) \in[0,1] \times[\tilde{T},+\infty)$.

Finally, using (2.9), (2.13), and (2.14), we get

$$
v(x, t) \geq C^{-1} e^{-2 \tilde{T}},
$$

for all $(x, t) \in[0,1] \times[0, \tilde{T}]$, which together with (2.17) implies

$$
v(x, t) \geq C_{0} \triangleq \min \left\{\frac{C^{-1} \alpha_{1}}{16}, C^{-1} e^{-2 \tilde{T}}\right\}
$$

for all $(x, t) \in[0,1] \times[0,+\infty)$. We finish the proof of Lemma 2.1]

To obtain the upper bound of $v$, we set

$$
M_{v}(t) \triangleq 1+\max _{x \in[0,1]} v(x, t)
$$

Then we have the following time-independent upper bound of $v$ for $\beta>0$. 
Lemma 2.2. For $\beta>0$, there exists a positive constant $C$ such that for all $(x, t) \in$ $[0,1] \times[0,+\infty)$,

$$
v(x, t) \leq C
$$

Proof. First, we claim that

$$
\int_{0}^{T} \max _{x \in[0,1]}\left(\theta^{\frac{1}{2}}(x, t)-2^{\frac{1}{2}}\right)_{+}^{2} d t \leq C .
$$

Indeed, on the one hand, for $\beta \in[1, \infty)$, we have

$$
\begin{aligned}
& \int_{0}^{T} \max _{x \in[0,1]}\left(\theta^{\frac{1}{2}}(x, t)-2^{\frac{1}{2}}\right)_{+}^{2} d t \\
& \leq C \int_{0}^{T} \max _{x \in[0,1]}\left(\theta^{\frac{\beta}{2}}(x, t)-2^{\frac{\beta}{2}}\right)_{+}^{2} d t \\
& \leq C \int_{0}^{T}\left(\int_{0}^{1}\left|\partial_{x}\left(\theta^{\frac{\beta}{2}}(x, t)-2^{\frac{\beta}{2}}\right)_{+}\right| d x\right)^{2} d t \\
& \leq C \int_{0}^{T} \int_{0}^{1} \frac{\theta^{\beta} \theta_{x}^{2}}{\theta^{2} v} d x \int_{0}^{1} v d x d t \\
& \leq C .
\end{aligned}
$$

On the other hand, for $\beta \in(0,1)$ and $\eta \triangleq(2-\beta) / 4 \in\left(0, \frac{1}{2}\right)$, integrating (2.10) multiplied by $\left(\theta^{\eta}-4^{\eta}\right)_{+} \theta^{\eta-1}$ over $(0,1) \times(0, T)$, we get

$$
\begin{aligned}
& \frac{\beta}{2} \int_{0}^{T} \int_{(\theta>4)(t)} \frac{\theta^{-1+\beta / 2} \theta_{x}^{2}}{v} d x d t \\
& \quad+\int_{0}^{T} \int_{\Omega} \frac{u_{x}^{2}+\left|\mathbf{w}_{x}\right|^{2}+\left|\mathbf{b}_{x}\right|^{2}}{v}\left(\theta^{\eta}-4^{\eta}\right)_{+} \theta^{\eta-1} d x d t \\
& =\frac{1}{2 \eta} \int_{\Omega}\left(\left(\theta^{\eta}-4^{\eta}\right)_{+}^{2}-\left(\theta_{0}^{\eta}-4^{\eta}\right)_{+}^{2}\right) d x+4^{\eta}(1-\eta) \int_{0}^{T} \int_{(\theta>4)(t)} \frac{\theta^{\beta} \theta_{x}^{2}}{v \theta^{2-\eta}} d x d t \\
& \quad+\int_{0}^{T} \int_{\Omega} \frac{\theta u_{x}}{v}\left(\theta^{\eta}-4^{\eta}\right)_{+} \theta^{\eta-1} d x d t \\
& \leq C(\varepsilon)+\varepsilon \int_{0}^{T} \int_{(\theta>4)(t)} \frac{\theta^{-1+\beta / 2} \theta_{x}^{2}}{v} d x d t+\frac{1}{2} \int_{0}^{T} \int_{\Omega} \frac{u_{x}^{2}}{v}\left(\theta^{\eta}-4^{\eta}\right)_{+} \theta^{\eta-1} d x d t,
\end{aligned}
$$

where in the last inequality we have used

$$
\begin{aligned}
& \int_{0}^{T} \int_{0}^{1} \frac{1}{v}\left(\theta^{\eta}-4^{\eta}\right)_{+} \theta^{\eta+1} d x d t \\
& \leq C \int_{0}^{T} \max _{x \in[0,1]}\left(\theta^{1 / 2}(x, t)-2^{1 / 2}\right)_{+}^{2} \int_{0}^{1} \theta^{2 \eta} d x d t \\
& \leq \varepsilon \int_{0}^{T} \int_{(\theta>4)(t)} \frac{\theta^{-1+\beta / 2} \theta_{x}^{2}}{v} d x d t+C(\varepsilon),
\end{aligned}
$$


due to

$$
\begin{aligned}
& \int_{0}^{T} \max _{x \in[0,1]}\left(\theta^{1 / 2}(x, t)-2^{1 / 2}\right)_{+}^{2} d t \\
& \leq C \int_{0}^{T} \int_{0}^{1} \frac{\theta_{x}^{2}}{\theta v} d x \int_{0}^{1} v d x d t \\
& \leq \varepsilon \int_{0}^{T} \int_{0}^{1} \frac{\theta^{-1+\beta / 2} \theta_{x}^{2}}{v} d x d t+C(\varepsilon) \int_{0}^{T} \int_{0}^{1} \frac{\theta^{-2+\beta} \theta_{x}^{2}}{v} d x d t \\
& \leq \varepsilon \int_{0}^{T} \int_{(\theta>4)(t)} \frac{\theta^{-1+\beta / 2} \theta_{x}^{2}}{v} d x d t+C(\varepsilon) .
\end{aligned}
$$

Combining (2.21) with (2.22) gives (2.19) for $\beta \in(0,1)$.

Next, it follows from (2.2) that

$$
\begin{aligned}
\left|\theta^{\frac{1}{2}}(x, t)-\bar{\theta}^{\frac{1}{2}}(t)\right| & \leq\left|\theta^{\frac{\beta+1}{2}}(x, t)-\bar{\theta}^{\frac{\beta+1}{2}}(t)\right| \\
& \leq \frac{\beta+1}{2}\left(\int_{0}^{1} \frac{\theta^{\beta} \theta_{x}^{2}}{\theta^{2} v} d x\right)^{1 / 2}\left(\int_{0}^{1} \theta v d x\right)^{1 / 2} \\
& \leq C V^{1 / 2}(t) M_{v}^{1 / 2}(t),
\end{aligned}
$$

which together with (2.12) leads to

$$
\theta(x, t) \leq C+C V(t) M_{v}(t)
$$

for all $(x, t) \in[0,1] \times[0, \infty)$.

Finally, standard calculations give

$$
\begin{aligned}
\max _{x \in[0,1]}|\mathbf{b}|^{2}(x, t) & \leq C \int_{0}^{1}\left|\mathbf{b} \cdot \mathbf{b}_{x}\right| d x \\
& \leq C \int_{0}^{1} \frac{\left|\mathbf{b}_{x}\right|^{2}}{v \theta} d x+C \int_{0}^{1} v \theta|\mathbf{b}|^{2} d x \\
& \leq C V(t)+C \max _{x \in[0,1]}\left(\theta^{1 / 2}(x, t)-2^{1 / 2}\right)_{+}^{2}+C,
\end{aligned}
$$

which together with (2.9), (2.13), (2.14), and (2.23) leads to

$$
\begin{aligned}
v(x, t) \leq & C+C \int_{0}^{t} e^{-\alpha_{1}(t-\tau)} \max _{x \in[0,1]}\left(\theta+v|\mathbf{b}|^{2}\right)(x, \tau) d \tau \\
\leq & C+C \int_{0}^{t} e^{-\alpha_{1}(t-\tau)}\left(1+(1+V(\tau)) M_{v}(\tau)\right) d \tau \\
& +C \int_{0}^{t} \max _{x \in[0,1]}\left(\theta^{1 / 2}(x, \tau)-2^{1 / 2}\right)_{+}^{2} M_{v}(\tau) d \tau .
\end{aligned}
$$

We thus obtain (2.18) from this, (2.11), (2.19), and the Gronwall inequality. The proof of Lemma 2.2 is finished.

For $\beta>0$, to obtain the time-independent lower bound of the temperature, we need the following uniform (with respect to time) estimate on the $L^{\infty}\left(0, T ; L^{p}\right)$-norm of $\theta^{-1}$.

Lemma 2.3. For $\beta>0$ and any $p>0$, there exists some positive constant $C(p)$ such that

$$
\sup _{\leq t \leq T} \int_{0}^{1} \theta^{-p} d x+\int_{0}^{T} \int_{0}^{1} \theta^{\beta-1-p} \theta_{x}^{2} d x d t \leq C(p) .
$$


Proof. It suffices to prove (2.25) for $p \neq 1$ since it holds for $p=1$ due to (2.11). Multiplying (2.10) by $1 / \theta^{p}$ and integration by parts gives

$$
\begin{aligned}
& \frac{1}{p-1}\left(\int_{0}^{1} \theta^{1-p} d x\right)_{t}+p \int_{0}^{1} \frac{\theta^{\beta} \theta_{x}^{2}}{v \theta^{p+1}} d x+\int_{0}^{1} \frac{u_{x}^{2}+\left|\mathbf{w}_{x}\right|^{2}+\left|\mathbf{b}_{x}\right|^{2}}{v \theta^{p}} d x \\
& =\int_{0}^{1} \frac{\left(\theta^{1-p}-1\right) u_{x}}{v} d x+\int_{0}^{1} \frac{u_{x}}{v} d x \\
& \leq C(p) \int_{0}^{1}\left|\theta^{\frac{1}{2}}-1\right|\left(\theta^{\frac{1}{2}-p}+1\right)\left|u_{x}\right| d x+\left(\int_{0}^{1} \ln v d x\right)_{t} \\
& \leq C(p) \max _{x \in[0,1]}\left|\theta^{\frac{1}{2}}-1\right|\left(\int_{0}^{1} \theta^{1-p} d x\right)^{1 / 2}\left(\int_{0}^{1} \frac{u_{x}^{2}}{v \theta^{p}} d x\right)^{1 / 2} \\
& \quad+\max _{x \in[0,1]}\left|\theta^{\frac{1}{2}}-1\right| \int_{0}^{1}\left|u_{x}\right| d x+\left(\int_{0}^{1} \ln v d x\right)_{t} \\
& \leq C(p) \max _{x \in[0,1]}\left(\theta^{\frac{1}{2}}-1\right)^{2}\left(1+\int_{0}^{1} \theta^{1-p} d x\right)+\frac{1}{2} \int_{0}^{1} \frac{u_{x}^{2}}{v \theta^{p}} d x \\
& \quad+C(p)\left(\int_{0}^{1}\left|u_{x}\right| d x\right)^{2}+\left(\int_{0}^{1} \ln v d x\right)_{t} .
\end{aligned}
$$

Next, it follows from (2.2) and (2.12) that

$$
\alpha_{1} \leq \int_{0}^{1} \theta d x \leq \int_{0}^{1}\left(\theta+\eta \frac{u^{2}+|\mathbf{w}|^{2}+v|\mathbf{b}|^{2}}{2}\right) d x \leq 1
$$

which yields that for any real number $q$,

$$
\begin{aligned}
\left|1-\bar{\theta}^{q}\right| & =\left|\int_{0}^{1} \frac{d}{d \eta}\left(\int_{0}^{1}\left(\theta+\eta \frac{u^{2}+|\mathbf{w}|^{2}+v|\mathbf{b}|^{2}}{2}\right) d x\right)^{q} d \eta\right| \\
& \leq C(q) \int_{0}^{1}\left(u^{2}+|\mathbf{w}|^{2}+v|\mathbf{b}|^{2}\right) d x \\
& \leq C(q) \max _{x \in[0,1]}(|u|+|\mathbf{w}|+|\mathbf{b}|)\left(\int_{0}^{1}\left(u^{2}+|\mathbf{w}|^{2}+v|\mathbf{b}|^{2}\right) d x\right)^{1 / 2} \\
& \leq C \int_{0}^{1}\left(\left|u_{x}\right|+\left|\mathbf{w}_{x}\right|+\left|\mathbf{b}_{x}\right|\right) d x \\
& \leq C\left(\int_{0}^{1} \frac{u_{x}^{2}+\left|\mathbf{w}_{x}\right|^{2}+\left|\mathbf{b}_{x}\right|^{2}}{v \theta} d x\right)^{1 / 2}\left(\int_{0}^{1} v \theta d x\right)^{1 / 2} \\
& \leq C V^{1 / 2}(t) .
\end{aligned}
$$

Then, it follows from (2.27) and (2.12) that for $\beta \in(0,1)$,

$$
\begin{aligned}
\max _{x \in[0,1]}\left|\theta^{\frac{1}{2}}-1\right| & \leq \max _{x \in[0,1]}\left|\theta^{\frac{1}{2}}-\bar{\theta}^{\frac{1}{2}}\right|+\max _{x \in[0,1]}\left|\bar{\theta}^{\frac{1}{2}}-1\right| \\
& \leq C \int_{0}^{1} \theta^{-\frac{1}{2}}\left|\theta_{x}\right| d x+C V^{1 / 2}(t) \\
& \leq C\left(\int_{0}^{1} \theta^{\beta-2} \theta_{x}^{2} d x\right)^{1 / 2}\left(\int_{0}^{1} \theta^{1-\beta} d x\right)^{1 / 2}+C V^{1 / 2}(t) \\
& \leq C V^{1 / 2}(t),
\end{aligned}
$$


and that for $\beta \geq 1$,

$$
\begin{aligned}
\max _{x \in[0,1]}\left|\theta^{\frac{1}{2}}-1\right| & \leq \max _{x \in[0,1]}\left|\theta^{\frac{1}{2}}-\bar{\theta}^{\frac{1}{2}}\right|+\max _{x \in[0,1]}\left|\bar{\theta}^{\frac{1}{2}}-1\right| \\
& \leq C \max _{x \in[0,1]}\left|\theta^{\frac{\beta}{2}}-\bar{\theta}^{\frac{\beta}{2}}\right|+C V^{1 / 2}(t) \\
& \leq C \int_{0}^{1} \theta^{\frac{\beta}{2}-1}\left|\theta_{x}\right| d x+C V^{1 / 2}(t) \\
& \leq C V^{1 / 2}(t) .
\end{aligned}
$$

It thus follows from (2.28), (2.29), and (2.11) that for $\beta>0$,

$$
\int_{0}^{T} \max _{x \in[0,1]}\left(\theta^{\frac{1}{2}}-1\right)^{2} d t \leq C
$$

Finally, noticing that for $p \in[0,1]$,

$$
\int_{0}^{1} \theta^{1-p} d x \leq \int_{0}^{1} \theta d x+1 \leq C
$$

and that both (2.11) and (2.2) imply that for $\beta \geq 0$,

$$
\sup _{0 \leq t<\infty} \int_{0}^{1}|\ln v| d x \leq C
$$

after using (2.27), (2.11), (2.30), and the Gronwall inequality, we obtain (2.25) from (2.26) and finish the proof of Lemma 2.3.

Lemma 2.4. For $\beta \geq 0$, there exists a positive constant $C$ such that for all $T>0$,

$$
\sup _{0 \leq t \leq T} \int_{0}^{1} v_{x}^{2} d x+\int_{0}^{T} \int_{0}^{1}\left((1+\theta) v_{x}^{2}+u_{x}^{2}+\left|\mathbf{b}_{x}\right|^{2}+\left|\mathbf{w}_{x}\right|^{2}\right) d x d t \leq C .
$$

Proof. Case $1(\beta=0)$. First, multiplying (1.3) by w and integrating the resulting equality over $(0,1)$ yields

$$
\begin{aligned}
& \frac{1}{2} \frac{d}{d t} \int_{0}^{1}|\mathbf{w}|^{2} d x+\int_{0}^{1} \frac{\left|\mathbf{w}_{x}\right|^{2}}{v} d x \\
& \leq C \int_{0}^{1}\left|\mathbf{w}_{x}\right||\mathbf{b}| d x \\
& \leq C \int_{0}^{1}\left|\mathbf{w}_{x}\right||\mathbf{b}|(\ln \theta-\ln \bar{\theta})_{+} d x+C \int_{0}^{1}\left|\mathbf{w}_{x}\right||\mathbf{b}|(1-\ln \theta)_{+} d x \\
& \leq C\left(\int_{0}^{1} \frac{\left|\mathbf{w}_{x}\right|^{2}}{v} d x\right)^{1 / 2}\left(\int_{0}^{1} v|\mathbf{b}|^{2} d x\right)^{1 / 2} \max _{x \in[0,1]}(\ln \theta-\ln \bar{\theta})_{+} \\
& +C\left(\int_{0}^{1} \frac{\left|\mathbf{w}_{x}\right|^{2}}{v \theta} d x\right)^{1 / 2} \max _{x \in[0,1]}\left(|\mathbf{b}|(\ln 2-\ln \theta)_{+}\right) \\
& \leq \frac{1}{2} \int_{0}^{1} \frac{\left|\mathbf{w}_{x}\right|^{2}}{v} d x+C V(t)
\end{aligned}
$$


where in the last inequality we have used the following two simple facts:

$$
\begin{aligned}
\max _{x \in[0,1]}(\ln \theta-\ln \bar{\theta})_{+} & \leq C \int_{0}^{1}\left|\left((\ln \theta-\ln \bar{\theta})_{+}\right)_{x}\right| d x \\
& \leq C\left(\int_{0}^{1} v d x \int_{0}^{1} \frac{\theta_{x}^{2}}{v \theta^{2}} d x\right)^{1 / 2} \\
& \leq C V^{1 / 2}(t),
\end{aligned}
$$

and

$$
\begin{aligned}
& \max _{x \in[0,1]}\left(|\mathbf{b}|(1-\ln \theta)_{+}\right) \\
& \leq C \int_{0}^{1}\left|\left(\mathbf{b}(1-\ln \theta)_{+}\right)_{x}\right| d x \\
& \leq C \int_{0}^{1}\left|\mathbf{b}_{x}\right| \theta^{-1 / 2} d x+C \int_{0}^{1}|\mathbf{b}| \frac{\left|\theta_{x}\right|}{\theta} d x \\
& \leq C\left(\int_{0}^{1} \frac{\mathbf{b}_{x}^{2}}{v \theta} d x \int_{0}^{1} v d x\right)^{1 / 2}+C\left(\int_{0}^{1} v|\mathbf{b}|^{2} d x \int_{0}^{1} \frac{\theta_{x}^{2}}{v \theta^{2}} d x\right)^{1 / 2} \\
& \leq C V^{1 / 2}(t) .
\end{aligned}
$$

Denoting

$$
\tilde{V}(t) \triangleq \int_{0}^{1} \frac{\left|\mathbf{w}_{x}\right|^{2}}{v} d x+V(t)
$$

We thus obtain from (2.33) and (2.11) that

$$
\int_{0}^{T} \tilde{V}(t) d t \leq C
$$

Next, using (1.1), we rewrite (1.2) as

$$
(\ln v)_{x t}=u_{t}+\left(\frac{\theta}{v}\right)_{x}+\mathbf{b} \cdot \mathbf{b}_{x}
$$

Adding (2.35) multiplied by $(\ln v)_{x}$ to (1.4) by $v \mathbf{b}$, and integrating the resulting equality over $(0,1) \times(0, T)$, one has

$$
\begin{aligned}
& \frac{1}{2} \frac{d}{d t} \int_{0}^{1}\left((\ln v)_{x}^{2}+v^{2}|\mathbf{b}|^{2}\right) d x+\int_{0}^{1}\left(\left|\mathbf{b}_{x}\right|^{2}+\frac{\theta}{v}(\ln v)_{x}^{2}\right) d x \\
& =\frac{d}{d t} \int_{0}^{1} u(\ln v)_{x} d x+\int_{0}^{1} \frac{u_{x}^{2}}{v} d x+\int_{0}^{1} \frac{\theta_{x}(\ln v)_{x}}{v} d x+\int_{0}^{1} v \mathbf{w}_{x} \cdot \mathbf{b} d x .
\end{aligned}
$$

Then, on the one hand, for the fourth term on the righthand side of (2.36), we have by (2.33),

$$
\begin{aligned}
\left|\int_{0}^{1} v \mathbf{w}_{x} \cdot \mathbf{b} d x\right| & \leq C M_{v}(t) \int_{0}^{1}\left|\mathbf{w}_{x}\right||\mathbf{b}| d x \\
& \leq C M_{v}(t) \tilde{V}(t) .
\end{aligned}
$$


On the other hand, for the third term on the righthand side of (2.36), integrating by part gives

$$
\begin{aligned}
\int_{0}^{1} \frac{\theta_{x}(\ln v)_{x}}{v} d x= & -\int_{0}^{1} \ln \frac{v}{C_{0}}\left(\frac{\theta_{x}}{v}\right)_{x} d x \\
= & -\int_{0}^{1} \ln \frac{v}{C_{0}}\left(\theta_{t}+\frac{\theta}{v} u_{x}-\frac{u_{x}^{2}+\left|\mathbf{w}_{x}\right|^{2}+\left|\mathbf{b}_{x}\right|^{2}}{v}\right) d x \\
= & -\left(\int_{0}^{1} \theta \ln \frac{v}{C_{0}} d x\right)_{t}+\int_{0}^{1} \frac{\theta u_{x}}{v} d x \\
& -\int_{0}^{1} \frac{\theta u_{x}}{v} \ln \frac{v}{C_{0}} d x+\int_{0}^{1} \frac{u_{x}^{2}+\left|\mathbf{w}_{x}\right|^{2}+\left|\mathbf{b}_{x}\right|^{2}}{v} \ln \frac{v}{C_{0}} d x .
\end{aligned}
$$

For the second term on the righthand side of (2.38), we have

$$
\begin{aligned}
\int_{0}^{1} \frac{\theta}{v} u_{x} d x & =\int_{0}^{1} \frac{(\theta-1)}{v} u_{x} d x+\int_{0}^{1} \frac{u_{x}}{v} d x \\
& \leq \varepsilon \int_{0}^{1} \frac{u_{x}^{2}}{v} d x+C(\varepsilon) \int_{0}^{1}(\theta-1)^{2} d x+\left(\int_{0}^{1} \ln v d x\right)_{t} \\
& \leq \varepsilon \int_{0}^{1} \frac{u_{x}^{2}}{v} d x+C(\varepsilon) V(t) M_{v}(t)+\left(\int_{0}^{1} \ln v d x\right)_{t},
\end{aligned}
$$

where in the last inequality we have used

$$
\begin{aligned}
\int_{0}^{1}(\theta-1)^{2} d x & \leq C \max _{x \in[0,1]}\left(\theta^{1 / 2}-1\right)^{2} \\
& \leq C \max _{x \in[0,1]}\left(\theta^{1 / 2}-\bar{\theta}^{1 / 2}\right)^{2}+C(1-\bar{\theta})^{2} \\
& \leq C\left(\int_{0}^{1}\left|\theta_{x}\right| \theta^{-1 / 2} d x\right)^{2}+C V(t) M_{v}(t) \\
& \leq C \int_{0}^{1} \frac{\theta_{x}^{2}}{\theta^{2} v} d x \int_{0}^{1} v \theta d x+C V(t) M_{v}(t) \\
& \leq C V(t) M_{v}(t)
\end{aligned}
$$

due to

$$
\begin{aligned}
1-\bar{\theta} & =\frac{1}{2} \int_{0}^{1}\left(u^{2}+|\mathbf{w}|^{2}+v|\mathbf{b}|^{2}\right) d x \\
& \leq C \int_{0}^{1}\left(\left|u_{x}\right|+\left|\mathbf{w}_{x}\right|+\left|\mathbf{b}_{x}\right|\right) d x \\
& \leq C\left(\int_{0}^{1} \frac{u_{x}^{2}+\left|\mathbf{w}_{x}\right|^{2}+\left|\mathbf{b}_{x}\right|^{2}}{v \theta} d x\right)^{1 / 2}\left(\int_{0}^{1} v \theta d x\right)^{1 / 2} \\
& \leq C V^{1 / 2}(t) M_{v}^{1 / 2}(t) .
\end{aligned}
$$

Similarly, for the third term on the righthand side of (2.38), we have

$$
\begin{aligned}
& -\int_{0}^{1} \frac{\theta}{v} u_{x} \ln \frac{v}{C_{0}} d x \\
& \leq C\left(\int_{0}^{1} \frac{u_{x}^{2}}{v} d x+V(t) M_{v}(t)\right) \ln M_{v}(t)-\frac{1}{2}\left(\int_{0}^{1} \ln ^{2} \frac{v}{C_{0}} d x\right)_{t},
\end{aligned}
$$


due to (2.39). Putting (2.39) and (2.42) into (2.38) gives

$$
\begin{aligned}
\int_{0}^{1} \frac{\theta_{x}(\ln v)_{x}}{v} d x \leq & \frac{d}{d t} \int_{0}^{1}\left(-\theta \ln \frac{v}{C_{0}}+\ln v-\frac{1}{2} \ln ^{2} \frac{v}{C_{0}}\right) d x \\
& +C\left(\int_{0}^{1} \frac{u_{x}^{2}+\left|\mathbf{b}_{x}\right|^{2}}{v} d x+\tilde{V}(t) M_{v}(t)\right) \ln M_{v}(t) .
\end{aligned}
$$

Putting (2.37) and (2.43) into (2.36) gives

$$
\begin{aligned}
& \frac{1}{2} \frac{d}{d t} \int_{0}^{1}\left((\ln v)_{x}^{2}+|v \mathbf{b}|^{2}+2 \theta \ln \frac{v}{C_{0}}+\ln ^{2} \frac{v}{C_{0}}-2 \ln v-2 u(\ln v)_{x}\right) d x \\
& +\int_{0}^{1}\left(\left|\mathbf{b}_{x}\right|^{2}+\frac{\theta}{v}(\ln v)_{x}^{2}\right) d x \\
& \leq C\left(\int_{0}^{1} \frac{u_{x}^{2}+\left|\mathbf{b}_{x}\right|^{2}}{v} d x+\tilde{V}(t) M_{v}(t)\right) \ln M_{v}(t) .
\end{aligned}
$$

Then, to estimate the first term on the righthand side of (2.44), we multiply (1.2) by $u$ and integrate the resultant equality over $(0,1)$ to get

$$
\frac{1}{2}\left(\int_{0}^{1} u^{2} d x\right)_{t}+\int_{0}^{1} \frac{u_{x}^{2}}{v} d x=\int_{0}^{1} \frac{\theta}{v} u_{x} d x-\int_{0}^{1} \mathbf{b} \cdot \mathbf{b}_{x} u d x .
$$

Thus, it follows from (2.41) and (2.11) that

$$
\begin{aligned}
\left|\int_{0}^{1} \mathbf{b} \cdot \mathbf{b}_{x} u d x\right| & \leq \max _{x \in[0,1]}|u|\left(\int_{0}^{1} v|\mathbf{b}|^{2} d x\right)^{1 / 2}\left(\int_{0}^{1} \frac{\left|\mathbf{b}_{x}\right|^{2}}{v} d x\right)^{1 / 2} \\
& \leq \varepsilon \int_{0}^{1} \frac{\left|\mathbf{b}_{x}\right|^{2}}{v} d x+C(\varepsilon) V(t) M_{v}(t)
\end{aligned}
$$

which together with (2.45) and (2.39) gives

$$
\begin{aligned}
& \frac{1}{2}\left(\int_{0}^{1} u^{2} d x\right)_{t}+\frac{1}{2} \int_{0}^{1} \frac{u_{x}^{2}}{v} d x \\
& \leq \frac{1}{4} \int_{0}^{1} \frac{\left|\mathbf{b}_{x}\right|^{2}}{v} d x+C V(t) M_{v}(t)+\left(\int_{0}^{1} \ln v d x\right)_{t} .
\end{aligned}
$$

Multiplying (1.4) by $\mathbf{b}$ and integrating the result over $(0,1)$, we obtain from (2.46) that

$$
\begin{aligned}
& \frac{1}{2}\left(\int_{0}^{1} v|\mathbf{b}|^{2} d x\right)_{t}+\int_{0}^{1} \frac{\left|\mathbf{b}_{x}\right|^{2}}{v} d x \\
& =\int_{0}^{1} \mathbf{b} \cdot \mathbf{b}_{x} u d x-\int_{0}^{1} \mathbf{w} \cdot \mathbf{b}_{x} d x \\
& \leq \frac{1}{4} \int_{0}^{1} \frac{\left|\mathbf{b}_{x}\right|^{2}}{v} d x+C V(t) M_{v}(t)+C \max _{x \in[0,1]}|\mathbf{w}|^{2} \\
& \leq \frac{1}{4} \int_{0}^{1} \frac{\left|\mathbf{b}_{x}\right|^{2}}{v} d x+C V(t) M_{v}(t)+C \int_{0}^{1} \frac{\left|\mathbf{w}_{x}\right|^{2}}{v} d x,
\end{aligned}
$$

which combined with (2.47) gives

$$
\begin{aligned}
& \left(\int_{0}^{1}\left(u^{2}+v|\mathbf{b}|^{2}\right) d x\right)_{t}+\int_{0}^{1} \frac{u_{x}^{2}+\left|\mathbf{b}_{x}\right|^{2}}{v} d x \\
& \leq C \tilde{V}(t) M_{v}(t)+2\left(\int_{0}^{1} \ln v d x\right)_{t} .
\end{aligned}
$$


Integrating this over $(0, t)$, we have by (2.31)

$$
\int_{0}^{t} \int_{0}^{1} \frac{u_{x}^{2}+\left|\mathbf{b}_{x}\right|^{2}}{v} d x d s \leq C+C \int_{0}^{t} \tilde{V}(s) M_{v}(s) d s .
$$

Next, it follows from (2.44) and (2.48) that

$$
\begin{aligned}
& \sup _{0 \leq s \leq t} \int_{0}^{1}(\ln v)_{x}^{2} d x+\int_{0}^{t} \int_{0}^{1}\left(\left|\mathbf{b}_{x}\right|^{2}+\frac{\theta}{v}(\ln v)_{x}^{2}\right) d x d s \\
& \leq C \ln \sup _{0 \leq s \leq t} M_{v}(s)+C \int_{0}^{t} \tilde{V}(s) M_{v}(s) d s \ln \sup _{0 \leq s \leq t} M_{v}(s) \\
& \leq C(\varepsilon)\left(1+\int_{0}^{t} \tilde{V}(s) M_{v}(s) d s\right) \ln \left(1+\int_{0}^{t} \tilde{V}(s) M_{v}(s) d s\right) \\
& \quad+C(\varepsilon)+\varepsilon \sup _{0 \leq s \leq t} M_{v}(s),
\end{aligned}
$$

where in the second inequality we have used

$$
f g \leq e^{f}-f-1+(1+g) \ln (1+g)-g, \text { for any } f \geq 0, g \geq 0,
$$

with

$$
f=\frac{1}{2} \ln \sup _{0 \leq s \leq t} M_{v}(s), \quad g=2 C \int_{0}^{t} \tilde{V}(s) M_{v}(s) d s .
$$

Then, direct computation shows

$$
\begin{aligned}
v-1 & \leq C\left(\int_{0}^{1} v^{2} d x\right)^{1 / 2}\left(\int_{0}^{1} \frac{v_{x}^{2}}{v^{2}} d x\right)^{1 / 2} \\
& \leq C M_{v}^{1 / 2}(t)\left(\int_{0}^{1} \frac{v_{x}^{2}}{v^{2}} d x\right)^{1 / 2}
\end{aligned}
$$

which gives

$$
M_{v}(t) \leq C+C \int_{0}^{1}(\ln v)_{x}^{2} d x .
$$

Combining this, (2.49), (2.34), and the Gronwall inequality shows that for any $(x, t) \in$ $[0,1] \times[0,+\infty)$,

$$
v(x, t) \leq C,
$$

which together with (2.49) and (2.34) implies

$$
\sup _{0 \leq t \leq T} \int_{0}^{1} v_{x}^{2} d x+\int_{0}^{T} \int_{0}^{1}\left(\theta v_{x}^{2}+u_{x}^{2}+\left|\mathbf{b}_{x}\right|^{2}+\left|\mathbf{w}_{x}\right|^{2}\right) d x d t \leq C .
$$

Finally, direct computation shows

$$
\begin{aligned}
\int_{0}^{1} v_{x}^{2} d x & =\int_{0}^{1} v_{x}^{2}(1-\theta) d x+\int_{0}^{1} v_{x}^{2} \theta d x \\
& \leq \int_{0}^{1} v_{x}^{2}(1-\theta)_{+} d x+\int_{0}^{1} v_{x}^{2} \theta d x \\
& \leq \frac{1}{2} \int_{0}^{1} v_{x}^{2} d x+C \max _{x \in[0,1]}\left(\theta^{1 / 2}-1\right)^{2}+\int_{0}^{1} v_{x}^{2} \theta d x
\end{aligned}
$$


where in the last inequality we have used (2.51). Combining this, (2.51), (2.40), and (2.50) gives (2.32) for $\beta=0$.

Case $2(\beta>0)$. First, we rewrite the momentum equation (1.2) as

$$
\left(u-\frac{v_{x}}{v}\right)_{t}=-\left(\frac{\theta}{v}+\frac{1}{2}|\mathbf{b}|^{2}\right)_{x} .
$$

Multiplying the above equation by $u-\frac{v_{x}}{v}$ and integrating the resultant equality yields that for any $t \in(0, T)$

$$
\begin{aligned}
\frac{1}{2} \int_{0}^{1}\left(u-\frac{v_{x}}{v}\right)^{2} d x-\frac{1}{2} \int_{0}^{1}\left(u-\frac{v_{x}}{v}\right)(x, 0) d x \\
=\int_{0}^{t} \int_{0}^{1}\left(\frac{\theta v_{x}}{v^{2}}-\frac{\theta_{x}}{v}-\mathbf{b} \cdot \mathbf{b}_{x}\right)\left(u-\frac{v_{x}}{v}\right) d x d t \\
=-\int_{0}^{t} \int_{0}^{1} \frac{\theta v_{x}^{2}}{v^{3}} d x d t+\int_{0}^{t} \int_{0}^{1} \frac{\theta u v_{x}}{v^{2}} d x d t \\
\quad-\int_{0}^{t} \int_{0}^{1} \frac{\theta x}{v}\left(u-\frac{v_{x}}{v}\right) d x d t-\int_{0}^{t} \int_{0}^{1} \mathbf{b} \cdot \mathbf{b}_{x}\left(u-\frac{v_{x}}{v}\right) d x d t \\
=-\int_{0}^{t} \int_{0}^{1} \frac{\theta v_{x}^{2}}{v^{3}} d x d t+\sum_{i=1}^{3} I_{i} .
\end{aligned}
$$

Each $I_{i}(i=1,2,3)$ can be estimated as follows:

First, Cauchy's inequality gives

$$
\begin{aligned}
\left|I_{1}\right| & \leq \frac{1}{4} \int_{0}^{t} \int_{0}^{1} \frac{\theta v_{x}^{2}}{v^{3}} d x d t+C \int_{0}^{T} \int_{0}^{1} u^{2} \theta d x d t \\
& \leq \frac{1}{4} \int_{0}^{t} \int_{0}^{1} \frac{\theta v_{x}^{2}}{v^{3}} d x d t+C,
\end{aligned}
$$

where we have used

$$
\begin{aligned}
\int_{0}^{T} \int_{0}^{1} u^{2} \theta d x d t & \leq C \int_{0}^{T} \int_{0}^{1} u^{2}\left(\left(\theta^{1 / 2}-1\right)^{2}+1\right) d x d t \\
& \leq C \int_{0}^{T}\left(\int_{0}^{1} u^{2} d x+\max _{x \in[0,1]}\left(\theta^{1 / 2}-1\right)^{2}\right) d t \\
& \leq C
\end{aligned}
$$

due to (2.27), (2.11), and (2.30).

Next, using (2.55), (2.11), and (2.25) with $p=\beta$, we have

$$
\begin{aligned}
\left|I_{2}\right| & \leq C \int_{0}^{T} \int_{0}^{1} u^{2} \theta d x d t+C \int_{0}^{T} \int_{0}^{1} \theta^{-1} \theta_{x}^{2} d x d t+\frac{1}{2} \int_{0}^{t} \int_{0}^{1} \frac{\theta v_{x}^{2}}{v^{3}} d x d t \\
& \leq C+\frac{1}{2} \int_{0}^{t} \int_{0}^{1} \frac{\theta v_{x}^{2}}{v^{3}} d x d t .
\end{aligned}
$$

Finally, integrating (2.10) over $(0,1) \times(0, T)$, we have by (2.31)

$$
\begin{aligned}
& \int_{0}^{T} \int_{0}^{1} \frac{u_{x}^{2}+\left|\mathbf{w}_{x}\right|^{2}+\left|\mathbf{b}_{x}\right|^{2}}{v} d x d t \\
& =\int_{0}^{1} \theta d x-\int_{0}^{1} \theta_{0} d x+\int_{0}^{T} \int_{0}^{1} \frac{\theta-1}{v} u_{x} d x+\int_{0}^{1} \ln v d x-\int_{0}^{1} \ln v_{0} d x \\
& \leq C+\frac{1}{2} \int_{0}^{T} \int_{0}^{1} \frac{u_{x}^{2}}{v} d x d t+C \int_{0}^{T} \max _{x \in[0,1]}\left(\theta^{1 / 2}-1\right)^{2} d t,
\end{aligned}
$$


which together with (2.30) gives

$$
\int_{0}^{T} \int_{0}^{1}\left(u_{x}^{2}+\left|\mathbf{w}_{x}\right|^{2}+\left|\mathbf{b}_{x}\right|^{2}\right) d x d t \leq C .
$$

Combining this with Cauchy's inequality leads to

$$
\begin{aligned}
\left|I_{3}\right| & \leq C \int_{0}^{t} \int_{0}^{1}\left(\left|\mathbf{b}_{x}\right|^{2}+|\mathbf{b}|^{2}\left(u-\frac{v_{x}}{v}\right)^{2}\right) d x d t \\
& \leq C+C \int_{0}^{t} V(t) \int_{0}^{1}\left(u-\frac{v_{x}}{v}\right)^{2} d x d t
\end{aligned}
$$

due to

$$
\max _{0 \leq x \leq 1}|\mathbf{b}|^{2} \leq C \int_{0}^{1} \frac{\left|\mathbf{b}_{x}\right|^{2}}{v \theta} d x \int_{0}^{1} v \theta d x \leq C V(t) .
$$

Putting (2.54), (2.56), and (2.58) into (2.53), we obtain after using the Gronwall inequality and (2.11) that

$$
\sup _{0 \leq t \leq T} \int_{0}^{1}\left(u-\frac{v_{x}}{v}\right)^{2} d x+\int_{0}^{T} \int_{0}^{1} \frac{\theta v_{x}^{2}}{v^{3}} d x d t \leq C,
$$

which together with (2.11) gives

$$
\sup _{0 \leq t \leq T} \int_{0}^{1} v_{x}^{2} d x+\int_{0}^{T} \int_{0}^{1} v_{x}^{2} \theta d x d t \leq C .
$$

Finally, since (2.52) still holds for $\beta>0$ due to (2.59), we have

$$
\int_{0}^{T} \int_{0}^{1} v_{x}^{2} d x d t \leq C
$$

which together with (2.59) and (2.57) proves (2.32) for $\beta>0$. The proof of Lemma 2.4 is finished.

Lemma 2.5. For $\beta \geq 0$, there is a positive constant $C$ such that for all $T>0$,

$$
\begin{aligned}
& \sup _{0 \leq t \leq T} \int_{0}^{1}\left(\left|\mathbf{b}_{x}\right|^{2}+\left|\mathbf{w}_{x}\right|^{2}\right) d x \\
& +\int_{0}^{T} \int_{0}^{1}\left(\left|\mathbf{b}_{t}\right|^{2}+\left|\mathbf{b}_{x x}\right|^{2}+\left|\mathbf{w}_{t}\right|^{2}+\left|\mathbf{w}_{x x}\right|^{2}\right) d x d t \leq C .
\end{aligned}
$$

Proof. First, rewriting (1.3) as

$$
\mathbf{w}_{t}=\frac{\mathbf{w}_{x x}}{v}-\frac{\mathbf{w}_{x} v_{x}}{v^{2}}+\mathbf{b}_{x},
$$

multiplying (2.61) by $\mathbf{w}_{x x}$, and integrating the resulting equality over $(0,1) \times(0, T)$, we obtain after using (1.9), (2.32), and Cauchy's inequality that

$$
\begin{aligned}
& \frac{1}{2} \int_{0}^{1}\left|\mathbf{w}_{x}\right|^{2} d x+\int_{0}^{T} \int_{0}^{1} \frac{\left|\mathbf{w}_{x x}\right|^{2}}{v} d x d t \\
& \leq C+\frac{1}{2} \int_{0}^{T} \int_{0}^{1} \frac{\left|\mathbf{w}_{x x}\right|^{2}}{v} d x d t+C \int_{0}^{T} \int_{0}^{1}\left(\left|\mathbf{b}_{x}\right|^{2}+\left|\mathbf{w}_{x}\right|^{2} v_{x}^{2}\right) d x d t \\
& \leq C+\frac{1}{2} \int_{0}^{T} \int_{0}^{1} \frac{\left|\mathbf{w}_{x x}\right|^{2}}{v} d x d t+C \int_{0}^{T} \max _{x \in[0,1]}\left|\mathbf{w}_{x}\right|^{2} d t .
\end{aligned}
$$


Noticing that for any $f \in\left\{\int_{0}^{1} f d x=0\right\} \cup\{f(0)=0\}$,

$$
\max _{x \in[0,1]} f^{2} \leq 2\left(\int_{0}^{1} f^{2} d x\right)^{1 / 2}\left(\int_{0}^{1} f_{x}^{2} d x\right)^{1 / 2},
$$

we get for any $\varepsilon>0$,

$$
\begin{aligned}
\int_{0}^{T} \max _{x \in[0,1]}\left|\mathbf{w}_{x}\right|^{2} d t & \leq C(\varepsilon) \int_{0}^{T} \int_{0}^{1}\left|\mathbf{w}_{x}\right|^{2} d x d t+\varepsilon \int_{0}^{T} \int_{0}^{1} \frac{\left|\mathbf{w}_{x x}\right|^{2}}{v} d x d t \\
& \leq C(\varepsilon)+\varepsilon \int_{0}^{T} \int_{0}^{1} \frac{\left|\mathbf{w}_{x x}\right|^{2}}{v} d x d t
\end{aligned}
$$

which combined with (2.62) leads to

$$
\sup _{0 \leq t \leq T} \int_{0}^{1}\left|\mathbf{w}_{x}\right|^{2} d x+\int_{0}^{T} \int_{0}^{1}\left|\mathbf{w}_{x x}\right|^{2} d x d t \leq C .
$$

Combining this, (2.61), (2.64), and (2.32) gives

$$
\begin{aligned}
\int_{0}^{T} \int_{0}^{1}\left|\mathbf{w}_{t}\right|^{2} d x d t & \leq C \int_{0}^{T} \int_{0}^{1}\left(\left|\mathbf{b}_{x}\right|^{2}+\left|\mathbf{w}_{x x}\right|^{2}+v_{x}^{2}\left|\mathbf{w}_{x}\right|^{2}\right) d x d t \\
& \leq C+C \int_{0}^{T} \max _{x \in[0,1]}\left|\mathbf{w}_{x}\right|^{2} d t \\
& \leq C .
\end{aligned}
$$

Next, rewriting (1.4) as

$$
\mathbf{b}_{t}=\frac{\mathbf{w}_{x}}{v}+\frac{\mathbf{b}_{x x}}{v^{2}}-\frac{\mathbf{b}_{x} v_{x}}{v^{3}}-\frac{\mathbf{b} u_{x}}{v},
$$

multiplying (2.67) by $\mathbf{b}_{x x}$ and integrating the result over $(0.1) \times(0, T)$, we deduce from (2.32), (2.11), (2.63), and Cauchy's inequality that

$$
\begin{aligned}
& \frac{1}{2} \int_{0}^{1}\left|\mathbf{b}_{x}\right|^{2} d x+\int_{0}^{T} \int_{0}^{1} \frac{\left|\mathbf{b}_{x x}\right|^{2}}{v^{2}} d x d t \\
& \leq C+\frac{1}{2} \int_{0}^{T} \int_{0}^{1} \frac{\left|\mathbf{b}_{x x}\right|^{2}}{v^{2}} d x d t+C \int_{0}^{T} \int_{0}^{1}\left(\left|\mathbf{b}_{x}\right|^{2} v_{x}^{2}+u_{x}^{2}|\mathbf{b}|^{2}+\left|\mathbf{w}_{x}\right|^{2}\right) d x d t \\
& \leq C+\frac{1}{2} \int_{0}^{T} \int_{0}^{1} \frac{\left|\mathbf{b}_{x x}\right|^{2}}{v^{2}} d x d t+C \int_{0}^{T} \max _{x \in[0,1]}\left|\mathbf{b}_{x}\right|^{2} d t+\max _{(x, t) \in[0,1] \times[0, T]}|\mathbf{b}|^{2} \\
& \leq C+\frac{3}{4} \int_{0}^{T} \int_{0}^{1} \frac{\left|\mathbf{b}_{x x}\right|^{2}}{v^{2}} d x d t+C \int_{0}^{T} \int_{0}^{1}\left|\mathbf{b}_{x}\right|^{2} d x d t \\
& \quad+C \sup _{0 \leq t \leq T} \int_{0}^{1}|\mathbf{b}|^{2} d x+\frac{1}{4} \sup _{0 \leq t \leq T} \int_{0}^{1}\left|\mathbf{b}_{x}\right|^{2} d x \\
& \leq C+\frac{3}{4} \int_{0}^{T} \int_{0}^{1} \frac{\left|\mathbf{b}_{x x}\right|^{2}}{v^{2}} d x d t+\frac{1}{4} \sup _{0 \leq t \leq T} \int_{0}^{1}\left|\mathbf{b}_{x}\right|^{2} d x
\end{aligned}
$$

which implies

$$
\sup _{0 \leq t \leq T} \int_{0}^{1}\left|\mathbf{b}_{x}\right|^{2} d x+\int_{0}^{T} \int_{0}^{1}\left|\mathbf{b}_{x x}\right|^{2} d x d t \leq C .
$$


Hence,

$$
\max _{(x, t) \in[0,1] \times[0, T]}|\mathbf{b}|^{2} \leq C+C \sup _{0 \leq t \leq T} \int_{0}^{1}\left|\mathbf{b}_{x}\right|^{2} d x \leq C .
$$

Finally, it follows from (2.67), (2.68), (2.32), and (2.69) gives

$$
\begin{aligned}
\int_{0}^{T} \int_{0}^{1}\left|\mathbf{b}_{t}\right|^{2} d x d t & \leq C \int_{0}^{T} \int_{0}^{1}\left(\left|\mathbf{b}_{x x}\right|^{2}+\left|\mathbf{b}_{x}\right|^{2} v_{x}^{2}+\left|\mathbf{w}_{x}\right|^{2}+|\mathbf{b}|^{2} u_{x}^{2}\right) d x d t \\
& \leq C+C \int_{0}^{T}\left(\max _{x \in[0,1]}\left|\mathbf{b}_{x}\right|^{2}+\int_{0}^{1} u_{x}^{2} d x\right) d t \\
& \leq C+C \int_{0}^{T} \int_{0}^{1}\left(\left|\mathbf{b}_{x}\right|^{2}+\left|\mathbf{b}_{x x}\right|^{2}\right) d x d t \\
& \leq C
\end{aligned}
$$

Combining this, (2.65), (2.66), and (2.68) gives (2.60) and finishes the proof of Lemma 2.5.

For further uses, we need the following estimate on the $L^{2}((0,1) \times(0, T))$-norm of $\theta_{x}$ for $\beta \in[0,1]$.

Lemma 2.6. If $\beta \in[0,1]$, there exists a positive constant $C$ such that for all $T>0$,

$$
\int_{0}^{T} \int_{0}^{1} \theta_{x}^{2} d x d t \leq C+C \int_{0}^{T}\left(\int_{0}^{1} u_{x}^{2} d x\right)^{2} d t .
$$

Proof. First, multiplying (2.10) by $\theta^{1-\frac{\beta}{2}}$ and integration by parts gives

$$
\begin{aligned}
& \frac{2}{4-\beta}\left(\int_{0}^{1} \theta^{2-\frac{\beta}{2}} d x\right)_{t}+\frac{(2-\beta)}{2} \int_{0}^{1} \frac{\theta^{\frac{\beta}{2}} \theta_{x}^{2}}{v} d x \\
& =-\int_{0}^{1} \frac{\theta^{2-\frac{\beta}{2}}}{v} u_{x} d x+\int_{0}^{1} \frac{\theta^{1-\frac{\beta}{2}}\left(u_{x}^{2}+\left|\mathbf{b}_{x}\right|^{2}+\left|\mathbf{w}_{x}\right|^{2}\right)}{v} d x \\
& =\int_{0}^{1} \frac{\left(\bar{\theta}^{2-\frac{\beta}{2}}-\theta^{2-\frac{\beta}{2}}\right)}{v} u_{x} d x+\left(1-\bar{\theta}^{2-\frac{\beta}{2}}\right) \int_{0}^{1} \frac{u_{x}}{v} d x \\
& \quad-\int_{0}^{1} \frac{u_{x}}{v} d x+\int_{0}^{1} \frac{\theta^{1-\frac{\beta}{2}}\left(u_{x}^{2}+\left|\mathbf{b}_{x}\right|^{2}+\left|\mathbf{w}_{x}\right|^{2}\right)}{v} d x \\
& \leq C \int_{0}^{1}\left|\theta^{2-\frac{\beta}{2}}-\bar{\theta}^{2-\frac{\beta}{2}}\right|\left|u_{x}\right| d x+C V(t)-\left(\int_{0}^{1} \ln v d x\right)_{t} \\
& \quad+\int_{0}^{1} \frac{\theta^{1-\frac{\beta}{2}}\left(u_{x}^{2}+\left|\mathbf{b}_{x}\right|^{2}+\left|\mathbf{w}_{x}\right|^{2}\right)}{v} d x
\end{aligned}
$$

where in the last inequality we have used (2.27). Direct calculations yield that for any 
$\delta>0$

$$
\begin{aligned}
& \int_{0}^{1}\left|\theta^{2-\frac{\beta}{2}}-\bar{\theta}^{2-\frac{\beta}{2}}\right|\left|u_{x}\right| d x \\
& \leq C \max _{x \in[0,1]}\left|\theta^{1-\frac{\beta}{4}}-\bar{\theta}^{1-\frac{\beta}{4}}\right|\left(\int_{0}^{1}\left(\theta^{2-\frac{\beta}{2}}+1\right) d x\right)^{1 / 2}\left(\int_{0}^{1} u_{x}^{2} d x\right)^{1 / 2} \\
& \leq \delta\left(\int_{0}^{1} \theta^{-\frac{\beta}{4}}\left|\theta_{x}\right| d x\right)^{2}+C(\delta) \int_{0}^{1}\left(\theta^{2-\frac{\beta}{2}}+1\right) d x \int_{0}^{1} u_{x}^{2} d x \\
& \leq C \delta \int_{0}^{1} \theta^{\beta / 2} \theta_{x}^{2} d x+C(\delta) V(t)+C(\delta) \int_{0}^{1}\left(\theta^{2-\frac{\beta}{2}}+1\right) d x \int_{0}^{1} u_{x}^{2} d x
\end{aligned}
$$

and that for any $\delta>0$

$$
\begin{aligned}
& \int_{0}^{1} \frac{\theta^{1-\frac{\beta}{2}} u_{x}^{2}}{v} d x \\
& \leq C\left(\max _{x \in[0,1]}\left|\theta^{1-\frac{\beta}{2}}-\bar{\theta}^{1-\frac{\beta}{2}}\right|+1\right) \int_{0}^{1} u_{x}^{2} d x \\
& \leq C \int_{0}^{1} \theta^{-\frac{\beta}{2}}\left|\theta_{x}\right| d x \int_{0}^{1} u_{x}^{2} d x+C \int_{0}^{1} u_{x}^{2} d x \\
& \leq \delta \int_{0}^{1}\left(\theta^{\beta-2}+\theta^{\frac{\beta}{2}}\right) \theta_{x}^{2} d x+C(\delta)\left(\int_{0}^{1} u_{x}^{2} d x\right)^{2}+C \int_{0}^{1} u_{x}^{2} d x .
\end{aligned}
$$

Putting (2.72) and (2.73) into (2.71), choosing $\delta$ suitably small, and using (2.11), (2.32), and the Gronwall inequality, one obtains

$$
\int_{0}^{1} \theta^{2-\beta / 2} d x+\int_{0}^{T} \int_{0}^{1} \theta^{\beta / 2} \theta_{x}^{2} d x d t \leq C+C \int_{0}^{T}\left(\int_{0}^{1} u_{x}^{2} d x\right)^{2} d t
$$

which together with (2.11) implies

$$
\begin{aligned}
\int_{0}^{T} \int_{0}^{1} \theta_{x}^{2} d x d t & \leq C \int_{0}^{T} \int_{0}^{1}\left(\theta^{\beta-2}+\theta^{\beta / 2}\right) \theta_{x}^{2} d x d t \\
& \leq C+C \int_{0}^{T} \int_{0}^{1} \theta^{\beta / 2} \theta_{x}^{2} d x d t \\
& \leq C+C \int_{0}^{T}\left(\int_{0}^{1} u_{x}^{2} d x\right)^{2} d t
\end{aligned}
$$

This gives (2.70) and finishes the proof of Lemma 2.6.

Lemma 2.7. For $\beta \geq 0$, there is a positive constant $C$ such that for all $T>0$,

$$
\sup _{0 \leq t \leq T} \int_{0}^{1} u_{x}^{2} d x+\int_{0}^{T} \int_{0}^{1}\left(u_{t}^{2}+u_{x x}^{2}+\theta_{x}^{2}\right) d x d t \leq C .
$$

Proof. First, rewriting (1.2) as

$$
u_{t}=\frac{u_{x x}}{v}-\frac{v_{x}}{v^{2}} u_{x}-\frac{\theta_{x}}{v}+\frac{\theta v_{x}}{v^{2}}-\mathbf{b} \cdot \mathbf{b}_{x},
$$


multiplying (2.75) by $u_{x x}$ and integrating the result over $(0,1)$, we have

$$
\begin{aligned}
& \frac{1}{2} \frac{d}{d t} \int_{0}^{1} u_{x}^{2} d x+\int_{0}^{1} \frac{u_{x x}^{2}}{v} d x \\
& \leq \frac{1}{2} \int_{0}^{1} \frac{u_{x x}^{2}}{v} d x+C \int_{0}^{1}\left(\theta_{x}^{2}+\theta^{2} v_{x}^{2}+|\mathbf{b}|^{2}\left|\mathbf{b}_{x}\right|^{2}+u_{x}^{2} v_{x}^{2}\right) d x .
\end{aligned}
$$

Direct computation yields that for any $\delta>0$,

$$
\begin{aligned}
& \int_{0}^{1}\left(\theta_{x}^{2}+\theta^{2} v_{x}^{2}+|\mathbf{b}|^{2}\left|\mathbf{b}_{x}\right|^{2}+u_{x}^{2} v_{x}^{2}\right) d x \\
& \leq C\left(\max _{x \in[0,1]} u_{x}^{2}+\max _{x \in[0,1]}(\theta-\bar{\theta})^{2}+1\right) \int_{0}^{1} v_{x}^{2} d x+C \int_{0}^{1}\left(\left|\mathbf{b}_{x}\right|^{2}+\theta_{x}^{2}\right) d x \\
& \leq C \max _{x \in[0,1]} u_{x}^{2}+C \max _{x \in[0,1]}(\theta-\bar{\theta})^{2}+C \int_{0}^{1}\left(v_{x}^{2}+\left|\mathbf{b}_{x}\right|^{2}\right) d x+C \int_{0}^{1} \theta_{x}^{2} d x \\
& \leq \delta \int_{0}^{1} u_{x x}^{2} d x+C(\delta) \int_{0}^{1} u_{x}^{2} d x+C \int_{0}^{1}\left(v_{x}^{2}+\left|\mathbf{b}_{x}\right|^{2}\right) d x+C \int_{0}^{1} \theta_{x}^{2} d x,
\end{aligned}
$$

where in the last inequality we have used (2.63). Putting (2.77) into (2.76) and choosing $\delta$ suitably small yields

$$
\int_{0}^{1} u_{x}^{2} d x+\int_{0}^{T} \int_{0}^{1} u_{x x}^{2} d x d t \leq C+C \int_{0}^{T} \int_{0}^{1} \theta_{x}^{2} d x d t
$$

due to (2.60) and (2.32).

Next, on the one hand, if $\beta>1$, choosing $p=\beta-1$ in (2.25) shows

$$
\int_{0}^{T} \int_{0}^{1} \theta_{x}^{2} d x d t \leq C
$$

which along with (2.78) gives

$$
\sup _{0 \leq t \leq T} \int_{0}^{1} u_{x}^{2} d x+\int_{0}^{T} \int_{0}^{1} u_{x x}^{2} d x d t+\int_{0}^{T} \int_{0}^{1} \theta_{x}^{2} d x d t \leq C .
$$

On the other hand, if $\beta \in[0,1]$, it follows from (2.78), (2.70), (2.32), and Gronwall's inequality that (2.80) still holds.

Finally, it follows from (2.75), (2.80), (2.77), and (2.32) that

$$
\int_{0}^{T} \int_{0}^{1} u_{t}^{2} d x d t \leq C
$$

which together with (2.80) gives (2.74) and finishes the proof of Lemma 2.7.

Lemma 2.8. For $\beta \geq 0$, there exists a positive constant $C$ such that for all $T>0$,

$$
\sup _{0 \leq t \leq T} \int_{0}^{1} \theta_{x}^{2} d x+\int_{0}^{T} \int_{0}^{1}\left(\theta_{t}^{2}+\theta_{x x}^{2}\right) d x d t \leq C .
$$


Proof. First, multiplying (2.10) by $\theta$ and integrating the result over $(0,1)$ yields

$$
\begin{aligned}
& \frac{1}{2} \frac{d}{d t} \int_{0}^{1} \theta^{2} d x+\int_{0}^{1} \frac{\theta^{\beta} \theta_{x}^{2}}{v} d x \\
& =-\int_{0}^{1} \frac{\theta^{2}-1}{v} u_{x} d x-\int_{0}^{1} \frac{u_{x}}{v} d x+\int_{0}^{1} \frac{\left(u_{x}^{2}+\left|\mathbf{w}_{x}\right|^{2}+\left|\mathbf{b}_{x}\right|^{2}\right) \theta}{v} d x \\
& \leq C \max _{x \in[0,1]}\left(u_{x}^{2}+\left|\mathbf{w}_{x}\right|^{2}+\left|\mathbf{b}_{x}\right|^{2}+(\theta-1)^{2}\right)-\frac{d}{d t} \int_{0}^{1} \ln v d x,
\end{aligned}
$$

where we have used (2.2). It follows from (2.41), (2.74), and (2.32) that

$$
\begin{aligned}
\int_{0}^{T} \max _{x \in[0,1]}(\theta-1)^{2} d t & \leq C \int_{0}^{T} \max _{x \in[0,1]}(\theta-\bar{\theta})^{2} d t+C \int_{0}^{T}(1-\bar{\theta})^{2} d t \\
& \leq C \int_{0}^{T} \int_{0}^{1} \theta_{x}^{2} d x d t+C \int_{0}^{T} V(t) d t \leq C,
\end{aligned}
$$

which together with (2.82), (2.60), and (2.74) gives

$$
\int_{0}^{T} \int_{0}^{1} \theta^{\beta} \theta_{x}^{2} d x d t \leq C .
$$

Next, noticing that integration by parts leads to

$$
\begin{aligned}
\int_{0}^{1} \theta^{\beta} \theta_{t}\left(\frac{\theta^{\beta} \theta_{x}}{v}\right)_{x} d x & =-\int_{0}^{1} \frac{\theta^{\beta} \theta_{x}}{v}\left(\theta^{\beta} \theta_{t}\right)_{x} d x \\
& =-\int_{0}^{1} \frac{\theta^{\beta} \theta_{x}}{v}\left(\theta^{\beta} \theta_{x}\right)_{t} d x \\
& =-\frac{1}{2} \int_{0}^{1} \frac{\left(\left(\theta^{\beta} \theta_{x}\right)^{2}\right)_{t}}{v} d x \\
& =-\frac{1}{2}\left(\int_{0}^{1} \frac{\left(\theta^{\beta} \theta_{x}\right)^{2}}{v} d x\right)_{t}-\frac{1}{2} \int_{0}^{1} \frac{\left(\theta^{\beta} \theta_{x}\right)^{2} u_{x}}{v^{2}} d x,
\end{aligned}
$$

multiplying (2.10) by $\theta^{\beta} \theta_{t}$ and integrating the resultant equality over $(0,1)$, we have

$$
\begin{aligned}
& \int_{0}^{1} \theta^{\beta} \theta_{t}^{2} d x+\frac{1}{2}\left(\int_{0}^{1} \frac{\left(\theta^{\beta} \theta_{x}\right)^{2}}{v} d x\right)_{t} \\
& =-\frac{1}{2} \int_{0}^{1} \frac{\left(\theta^{\beta} \theta_{x}\right)^{2} u_{x}}{v^{2}} d x+\int_{0}^{1} \frac{\theta^{\beta} \theta_{t}\left(-\theta u_{x}+u_{x}^{2}+\left|\mathbf{w}_{x}\right|^{2}+\left|\mathbf{b}_{x}\right|^{2}\right)}{v} d x \\
& \leq C \max _{x \in[0,1]}\left(\left|u_{x}\right| \theta^{\beta / 2}\right) \int_{0}^{1} \theta^{3 \beta / 2} \theta_{x}^{2} d x+\frac{1}{2} \int_{0}^{1} \theta^{\beta} \theta_{t}^{2} d x+C \int_{0}^{1} \theta^{\beta+2} u_{x}^{2} d x \\
& \quad+C \int_{0}^{1} \theta^{\beta}\left(u_{x}^{4}+\left|\mathbf{w}_{x}\right|^{4}+\left|\mathbf{b}_{x}\right|^{4}\right) d x \\
& \leq C \int_{0}^{1} \theta^{2 \beta} \theta_{x}^{2} d x \int_{0}^{1} \theta^{\beta} \theta_{x}^{2} d x+\frac{1}{2} \int_{0}^{1} \theta^{\beta} \theta_{t}^{2} d x+\max _{x \in[0,1]}\left(\theta^{1+3 \beta / 4}-\bar{\theta}^{1+3 \beta / 4}\right)^{4} \\
& \quad+C\left(1+\int_{0}^{1} \theta^{2 \beta} \theta_{x}^{2} d x\right) \max _{x \in[0,1]}\left(u_{x}^{2}+u_{x}^{4}+\left|\mathbf{w}_{x}\right|^{4}+\left|\mathbf{b}_{x}\right|^{4}\right)
\end{aligned}
$$

due to

$$
\max _{x \in[0,1]}\left(\theta^{\beta+1}-\bar{\theta}^{\beta+1}\right)^{2} \leq C \int_{0}^{1} \theta^{2 \beta} \theta_{x}^{2} d x
$$


Next, combining (2.63) and (2.74) gives

$$
\begin{aligned}
\int_{0}^{T} \max _{x \in[0,1]} u_{x}^{4} d t & \leq C \int_{0}^{T} \int_{0}^{1} u_{x}^{2} d x \int_{0}^{1} u_{x x}^{2} d x d t \\
& \leq C \int_{0}^{T} \int_{0}^{1} u_{x x}^{2} d x d t \leq C .
\end{aligned}
$$

Using (2.60) and applying similar arguments to $\mathbf{b}$ and $\mathbf{w}$ implies

$$
\int_{0}^{T} \max _{x \in[0,1]}\left|\mathbf{b}_{x}\right|^{4} d t \leq C, \quad \int_{0}^{T} \max _{x \in[0,1]}\left|\mathbf{w}_{x}\right|^{4} d t \leq C .
$$

Noticing that

$$
\begin{aligned}
\max _{x \in[0,1]}\left(\theta^{1+3 \beta / 4}-\bar{\theta}^{1+3 \beta / 4}\right)^{4} & \leq C\left(\int_{0}^{1} \theta^{3 \beta / 4}\left|\theta_{x}\right| d x\right)^{4} \\
& \leq C \int_{0}^{1} \theta^{2 \beta} \theta_{x}^{2} d x \int_{0}^{1} \theta^{\beta} \theta_{x}^{2} d x
\end{aligned}
$$

we deduce from (2.84), (2.83), (2.86) $-(2.88)$, and the Gronwall inequality that

$$
\sup _{0 \leq t \leq T} \int_{0}^{1}\left(\theta^{\beta} \theta_{x}\right)^{2} d x+\int_{0}^{T} \int_{0}^{1} \theta^{\beta} \theta_{t}^{2} d x d t \leq C,
$$

which together with (2.85) in particular gives

$$
\max _{(x, t) \in[0,1] \times[0, T]} \theta(x, t) \leq C .
$$

Next, it follows from (2.74) and (2.90) that

$$
\begin{aligned}
\int_{0}^{T} \int_{0}^{1}\left(\theta^{\beta+1}-\bar{\theta}^{\beta+1}\right)^{2} d x d t & \leq C \int_{0}^{T}\left(\int_{0}^{1} \theta^{\beta}\left|\theta_{x}\right| d x\right)^{2} d t \\
& \leq C \int_{0}^{T} \int_{0}^{1} \theta_{x}^{2} d x d t \leq C,
\end{aligned}
$$

which together with (2.90), (2.89), and (2.10) shows

$$
\begin{aligned}
& \int_{0}^{T}\left|\frac{d}{d t} \int_{0}^{1}\left(\theta^{\beta+1}-\bar{\theta}^{\beta+1}\right)^{2} d x\right| d t \\
& \leq C \int_{0}^{T} \int_{0}^{1}\left(\theta^{\beta+1}-\bar{\theta}^{\beta+1}\right)^{2} d x d t+C \int_{0}^{T} \int_{0}^{1}\left(\theta^{\beta} \theta_{t}^{2}+\bar{\theta}_{t}^{2}\right) d x d t \\
& \leq C+C \int_{0}^{T} \int_{0}^{1}\left(u_{x}^{2}+\left|\mathbf{b}_{x}\right|^{2}+\left|\mathbf{w}_{x}\right|^{2}\right) d x d t \\
& \leq C .
\end{aligned}
$$

Thus, both (2.91) and (2.92) lead to

$$
\lim _{t \rightarrow \infty} \int_{0}^{1}\left(\theta^{\beta+1}-\bar{\theta}^{\beta+1}\right)^{2} d x=0
$$


which together with (2.89) gives

$$
\begin{aligned}
\max _{x \in[0,1]}\left(\theta^{\beta+1}-\bar{\theta}^{\beta+1}\right)^{4} & \leq C \int_{0}^{1}\left(\theta^{\beta+1}-\bar{\theta}^{\beta+1}\right)^{2} d x \int_{0}^{1} \theta^{2 \beta} \theta_{x}^{2} d x \\
& \leq C \int_{0}^{1}\left(\theta^{\beta+1}-\bar{\theta}^{\beta+1}\right)^{2} d x \rightarrow 0 \text { as } t \rightarrow \infty .
\end{aligned}
$$

It thus follows from (2.93) and (2.12) that there exists some $T_{0}>0$ such that

$$
\theta(x, t) \geq \alpha_{1} / 2
$$

for all $(x, t) \in[0,1] \times\left[T_{0}, \infty\right)$. Moreover, it follows from [16, 21] that there exists some constant $C \geq 2 / \alpha_{1}$ such that

$$
\theta(x, t) \geq C^{-1}
$$

for all $(x, t) \in[0,1] \times\left[0, T_{0}\right]$. Combining this, (2.94), and (2.90) yields that for all $(x, t) \in[0,1] \times[0, \infty)$,

$$
C^{-1} \leq \theta(x, t) \leq C
$$

which together with (2.89) gives

$$
\sup _{0 \leq t \leq T} \int_{0}^{1} \theta_{x}^{2} d x+\int_{0}^{T} \int_{0}^{1} \theta_{t}^{2} d x d t \leq C .
$$

Finally, it follows from (2.10) that

$$
\frac{\theta^{\beta} \theta_{x x}}{v}=-\frac{\beta \theta^{\beta-1} \theta_{x}^{2}}{v}+\frac{\theta^{\beta} \theta_{x} v_{x}}{v^{2}}-\frac{u_{x}^{2}+\left|\mathbf{b}_{x}\right|^{2}+\left|\mathbf{w}_{x}\right|^{2}}{v}+\frac{\theta u_{x}}{v}+\theta_{t},
$$

which together with (2.95), (2.32), (2.86), (2.87), (2.74), (2.96), and (2.63) yields

$$
\begin{aligned}
\int_{0}^{T} \int_{0}^{1} \theta_{x x}^{2} d x d t & \leq C \int_{0}^{T} \int_{0}^{1}\left(\theta_{x}^{4}+\theta_{x}^{2} v_{x}^{2}+u_{x}^{4}+\left|\mathbf{b}_{x}\right|^{4}+\left|\mathbf{w}_{x}\right|^{4}+u_{x}^{2}+\theta_{t}^{2}\right) d x d t \\
& \leq C+C \int_{0}^{T} \max _{x \in[0,1]} \theta_{x}^{2} d t \\
& \leq C+\frac{1}{2} \int_{0}^{T} \int_{0}^{1} \theta_{x x}^{2} d x d t .
\end{aligned}
$$

Combining this with (2.96) proves (2.81) and finishes the proof of Lemma 2.8.

Finally, we have the following nonlinearly exponential stability of the strong solutions.

Lemma 2.9. There exist some positive constants $C$ and $\eta_{0}$ both depending only on $\beta,\left\|\left(v_{0}, u_{0}, \theta_{0}, \mathbf{b}_{0}, \mathbf{w}_{0}\right)\right\|_{H^{1}(0,1)}, \inf _{x \in[0,1]} v_{0}(x)$, and $\inf _{x \in[0,1]} \theta_{0}(x)$ such that

$$
\|(v-1, u, \theta-1, \mathbf{b}, \mathbf{w})(\cdot, t)\|_{H^{1}(0,1)} \leq C e^{-\eta_{0} t}
$$

Proof. Noticing that all the constants $C$ in Lemmas 2.4, 2.5, 2.7, and 2.8 are independent of $T$, we have

$$
\int_{0}^{\infty}\left|\frac{d}{d t}\left(\left\|v_{x}\right\|_{L^{2}}^{2},\left\|u_{x}\right\|_{L^{2}}^{2},\left\|\theta_{x}\right\|_{L^{2}}^{2},\left\|\mathbf{b}_{x}\right\|_{L^{2}}^{2},\left\|\mathbf{w}_{x}\right\|_{L^{2}}^{2}\right)\right| d t \leq C,
$$


where we have used

$$
\int_{0}^{1} u_{x} u_{x t} d x=-\int_{0}^{1} u_{t} u_{x x} d x .
$$

It thus follows from (2.98), (2.32), and (2.81) that

$$
\lim _{t \rightarrow \infty}\left\|\left(v_{x}, u_{x}, \theta_{x}, \mathbf{b}_{x}, \mathbf{w}_{x}\right)(\cdot, t)\right\|_{L^{2}(0,1)}=0,
$$

which in particular implies

$$
\lim _{t \rightarrow \infty}\|(v-1, u, \theta-1, \mathbf{b}, \mathbf{w})(\cdot, t)\|_{H^{1}(0,1)}=0 .
$$

With (2.99) at hand, the proof of (2.97) is standard (c.f. [29]).

\section{References}

[1] Amosov, A. A., Zlotnik, A. A. Global generalized solutions of the equations of the one-dimensional motion of a viscous heat-conducting gas. Soviet Math. Dokl. 38 (1989), 1-5.

[2] Amosov A. A. and A. A. Zlotnik, A difference scheme on a non-uniform mesh for the equations of one-dimensional magnetic gas dynamics. U.S.S.R. Compu. Maths. Math. Phys., 29 (1989), 129-139.

[3] Amosov, A. A., Zlotnik, A. A. Solvability "in the large" of a system of equations of the one-dimensional motion of an inhomogeneous viscous heat-conducting gas. Math. Notes 52 (1992),753-763.

[4] Amosov, A. A., Zlotnik, A. A. On the stability of generalizedsolutions of equations of one-dimensional motion of a viscous heat-conducting gas, Sib. Math. J. 38 (1997) 663-684.

[5] Antontsev, S. N., Kazhikhov, A. V., Monakhov, V. N. Boundary Value Problems in Mechanics of Nonhomogeneous Fluids. Amsterdam, New York: North-Holland, 1990 .

[6] Cabannes, H.: Theoretical Magnetofluiddynamics. Academic Press, New York (1970)

[7] Chen, G. Q., Wang, D. H.: Global solutions for nonlinear magnetohydrodynamics with large initial data. J. Differ. Equ., 182 (2002), 344-376.

[8] Chapman, S., Colwing, T. G. The mathematical theory of nonuniform gases Cambridge Mathematical Library. 3rd edn. New York: Springer (1994)

[9] Chen, G. Q., Wang, D. H.: Existence and continuous dependence of large solutions for the magnetohydrodynamics equations. Z. Angew. Math. Phys., 54 (2003), 608632 .

[10] Duan, R., Jiang, F., Jiang, S.: On the Rayleigh Taylor instability for incompressible, inviscid magnetohydrodynamic flows. SIAM J. Appl. Math., 71 (2011) 1990-2013. 
[11] Fan, J. S., Huang, S. X., Li, F. C.: Global strong solutions to the planar compressible magnetohydrodynamic equations with large initial data and vaccum. Kinetic \& Related Models, 10 (2017), 1035-1053.

[12] Fan, J. S., Jiang, S., Nakamura, G.: Vanishing shear viscosity limit in the magnetohydrodynamics equations. Commun. Math. Phys., 270 (2007), 691-708.

[13] Hoff, D., Tsyganov, E.: Uniqueness and continuous dependence of weak solutions in compressible magnetohydrodynamics. Z. Angew. Math. Phys., 56 (2005), 791804.

[14] Hu,Y., Ju, Q.: Global large solutions of magnetohydrodynamics with temperaturedependent heat conductivity.Z. Angew. Math. Phys., 66 (2015), 865-889.

[15] Huang, B., Shi X. D.: Nonlinearly exponential stability of compressible NavierStokes system with degenerate heat-conductivity. J. Differ. Equ., in press. doi.org/10.1016/j.jde.2019.09.006

[16] Huang, B., Shi X. D., Sun Y.: Global strong solutions to magnetohydrodynamics with density-dependent viscosity and degenerate heat-conductivity. Nonlinearity, 32 (2019), 4395-4412.

[17] Jeffrey, A., Taniuti, T.: Non-Linear Wave Propagation. With Applications to Physics and Magnetohydrodynamics. Academic Press, New York (1964)

[18] Jenssen, H. K., Karper, T. K.: One-dimensional compressible flow with temperature de- pendent transport coefficients. SIAM Journal on Mathematical Analysis, 42 (2010), 904-930.

[19] Jiang, F., Jiang, S., Wang, Y.J.: On the Rayleigh-Taylor instability for incompressible viscous magnetohydrodynamic equations. Commun. Partial Differ. Equ., 39 (2014), 399-438.

[20] Kazhikhov, A. V. To a theory of boundary value problems for equations of onedimensional nonstationary motion of viscous heat-conduction gases, in: Boundary Value Problems for Hydrodynamical Equations, No. 50, Institute of Hydrodynamics, Siberian Branch Acad. USSR, 1981, pp. 37-62, in Russian.

[21] Kazhikhov, A. V.: A priori estimates for the solutions of equations of magnetic gas dynamics, Boundary value problems for equations of mathematical physics, Krasnoyarsk, 1987. In Russian

[22] Kazhikhov, A. V., Shelukhin, V. V.: Unique global solution with respect to time of initial boundary value problems for one-dimensional equations of a viscous gas. J. Appl. Math. Mech., 41 (1977), 273-282.

[23] Kulikovskiy, A. G., Lyubimov, G. A.: Magnetohydrodynamics. Addison-Wesley, Reading (1965)

[24] Laudau, L. D., Lifshitz, E. M.: Electrodynamics of Continuous Media. 2nd edn. Pergamon, New York (1984)

[25] Nagasawa, T. On the one-dimensional motion of the polytropic ideal gas non-fixed on the boundary. J. Diff. Eqs. 65 (1986), 49-67. 
[26] Nagasawa, T. On the asymptotic behavior of the one-dimensional motion of the polytropic ideal gas with stress-free condition. Quart. Appl. Math. 46 (1988), 665679.

[27] Nagasawa, T. On the one-dimensional free boundary problem for the heatconductive compressible viscous gas. In: Mimura, M., Nishida, T. (eds.) Recent Topics in Nonlinear PDE IV, Lecture Notes in Num. Appl. Anal. 10, Amsterdam, Tokyo: Kinokuniya/North-Holland, 1989, pp. 83-99.

[28] Nishida, T. Equations of motion of compressible viscous fluids. In: Nishida, T., Mimura, M., Fujii, H. (eds.) Pattern and Waves, Amsterdam, Tokyo: Kinokuniya/North-Holland, 1986, pp. 97-128.

[29] Okada, M.; Kawashima, S. On the equations of one-dimensional motion of compressible viscous fluids, J. Math. Kyoto Univ., 23 (1983), 55-71.

[30] Pan, R. H., Zhang, W. Z.: Compressible Navier-Stokes equations with temperature dependent heat conductivities. Commun. Math. Sci., 13(2015), 401-425.

[31] Polovin, R. V., Demutskii, V. P.: Fundamentals of Magnetohydrodynamics. Consultants Bureau, New York (1990)

[32] Qin, Y. Nonlinear Parabolic-Hyperbolic Coupled Systems and Their Attractors, Operator Theory, Advances and Applications, Vol 184. Basel, Boston, Berlin: Birkhäuser, 2008

[33] Wang, D. H.: Large solutions to the initial-boundary value problem for planar magnetohydrodynamics. SIAM J. Appl. Math., 63 (2003), 1424-1441. 